\title{
PRKACA somatic mutations are rare findings in aldosterone-producing adenomas
}

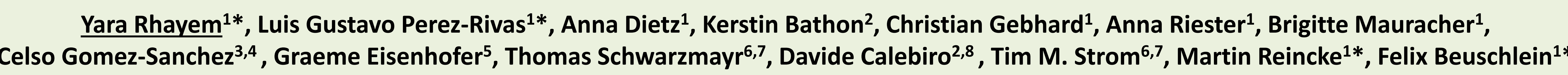

Department of Endocrine Research, Medizinische Klinik und Poliklinik IV, Klinikum der Universität München, Ludwig-Maximilians-Universität München, Munich, Germany ; ${ }^{2}$ Institute of Pharmacology and Toxicology, University of Würzburg, Würzburg, Germany ; Division of Endocrinology, G.V. (Sonny) Menticis

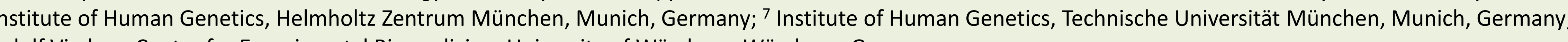
*YR, LGP-R, MR and FB contributed equally to this work.

\section{Introduction}

Primary aldosteronism (PA) is the predominant endocrine cause of secondary hypertension, affecting $5-10 \%$ of hypertensive producing adenomas (APA) and bilateral adrenal herperstant hypertension. The two predominant causes of PA are aldosteroneassociated with hypokalemia. So far, at least five candidate genes are implicated in PA: KCNJ5, CACNA1D, ATP1A1, ATP2B3, and CACNA1H (1-5), mutations result in electrophysiological alterations, consecutive increase in intracellular calcium levels and ultimately increase in the expression of CYP11B2, which encodes aldosterone synthase required for aldosterone biosynthesis. Another key activator for adrenocortical steroidogenesis and cell growth is cyclic AMP (CAMP), a second messenger, whic subunit $(C \alpha)$, have been reported in adenomas of the adrenal cortex $(6-10)$. In particular, the most frequent of the (p.Leu206Arg) was found to be restricted to cortisol producing adenomas (CPA) associated with overt Cushing's syndrome. Although aldosterone- and cortisol co-secreting adenoma and subclinical Cushing's syndrome can occur in PA patients $(11,12)$ the molecular causes for steroid co-secretion have remained uncertain. We report on in depth investigation of two cases of $P A$ presenting with somatic mutations of PRKACA identified by exome sequencing and evaluated for their clinical and molecul phenotypes.

Results

Table1: Clinical and biochemical parameters of the PA-patients with PRKACA somatic mutations.

Somatic PRKACA mutations were identified by exome sequencing in 2/122 (1.6\%) cases described in Table 1. Case 1 presented c.262C>G (p.His88Asp) mutation in exon 4 while a c.617A>C (p.Leu206Arg) mutation in exon 7 was identified in case 2 (Fig.1A). His88 is the only residue in the small lobe of the conserved catalytic core of the C subunit of PKA to interact with the phosphate
on residue Thr198, the essential phosphorylation site on the surface of the large lobe (Fig.1B). Situated at the cleft interface, on residue Thr198, the essential phosphorylation site on the surface of the large lobe (Fig.1B). Situated at the cleft interface,
His88 was found to complement Ser100 at the auto-inhibitor sequence P+2 in the type I regulatory subunit and therefore Hiss8 is Hiss8 was found to complement Sertoo at the auto-inhibitor sequence P+2 in the type I regulatory subunit and therefore Hiss8 is
also involved in the interaction with the Rla subunit of PKA. Measurement of PKA catalytic activity demonstrated that mutated His88Asp Ca subunit of PKA resulted in a significantly lower enzymatic PKA activity in comparison to the wild-type enzyme when co-transfected with either Rla or with RUB in HEK293 cells, both in presence and in absence of CAMP (Fig 1C). On the contran mutated Leu206Arg resulted in a consititutive elevated activity not suppressed by any of the regulatory subunits, as previously described (6). The APA resected from the two cases were of similar size $(0.9 \mathrm{vs} 1.2 \mathrm{~cm})$ and both were composed of zona fasciculata and zona glomerulosa-like cells. Double immunofluorescence analysis (Fig. 2A) showed CYP11B2 expression and, to a lesser extent, CYP11B1 in case 1; conversely, the adenoma cells in case 2 were mostly positive for CYP11B1. Multi-steroid analysis with the multi-steroid fingerprint of patients carrying KCNIS somatic mutations (Fig.2B).

Figure 1: Identification and functional characterization of PRKACA variants

$$
\begin{aligned}
& \begin{array}{l}
\text { case } 1 \\
c .262 C \rightarrow G \text {, (p. His88Asp) }
\end{array} \\
& \text { blood } \\
& \text { tumor MWWhlow }
\end{aligned}
$$
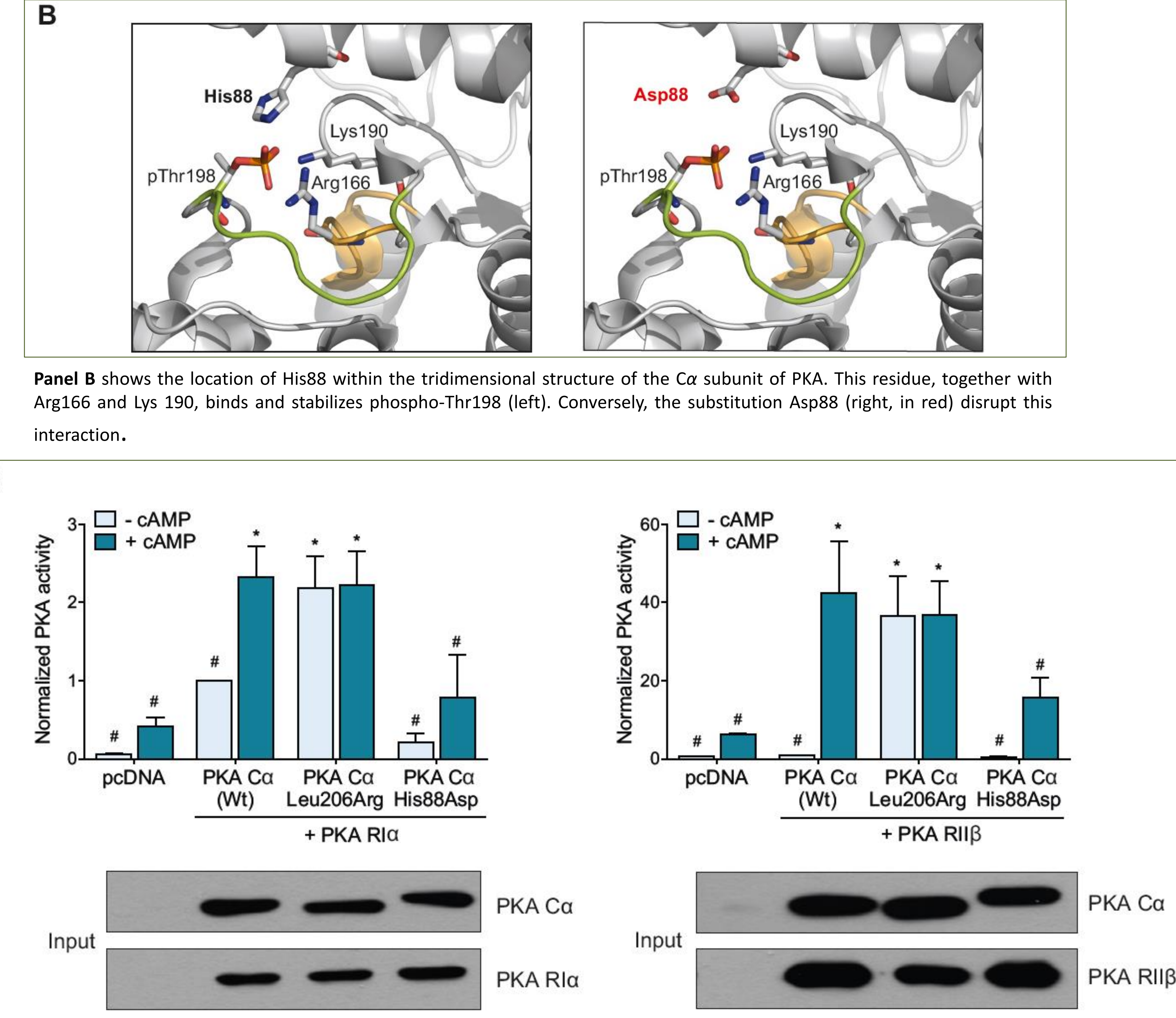

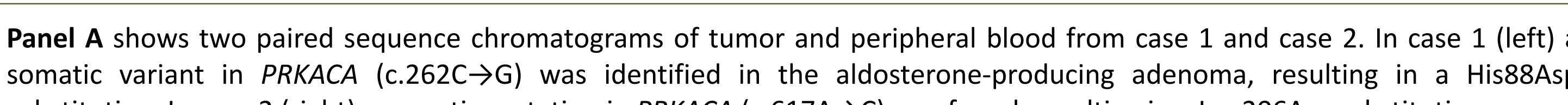

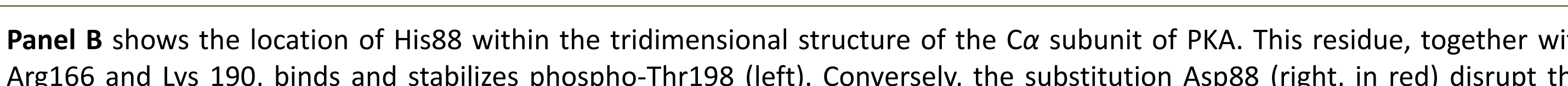

\section{Materials and Methods}

Patients were diagnosed with PA according to institutional and Endocrine Society Clinical Practice Guidelines and were included in the German Conn's Registry. Baseline clinical characterization included multi-steroid analysis of peripheral blood samples. APA tissues witiation was done by cross-sectional imaging (MRI) and adrenal venous sampling in PA patients. Klinikum der Universität München. Surgically resected adrenocortical tissues were examined by a clinical pathologist.

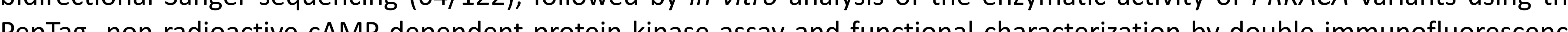
of CYP11B2 and CYP11B1 expression in the corresponding tumor tissues. All patients provided written informed consent and the study was approved by the ethics committee of the Ludwig-Maximilian University of Munich. Biochemical and clinical data were prospectively collected. dentification of PRKACA somatic variants in APA was performed in the 122 APA by whole-exome sequencing (58/122) or direct

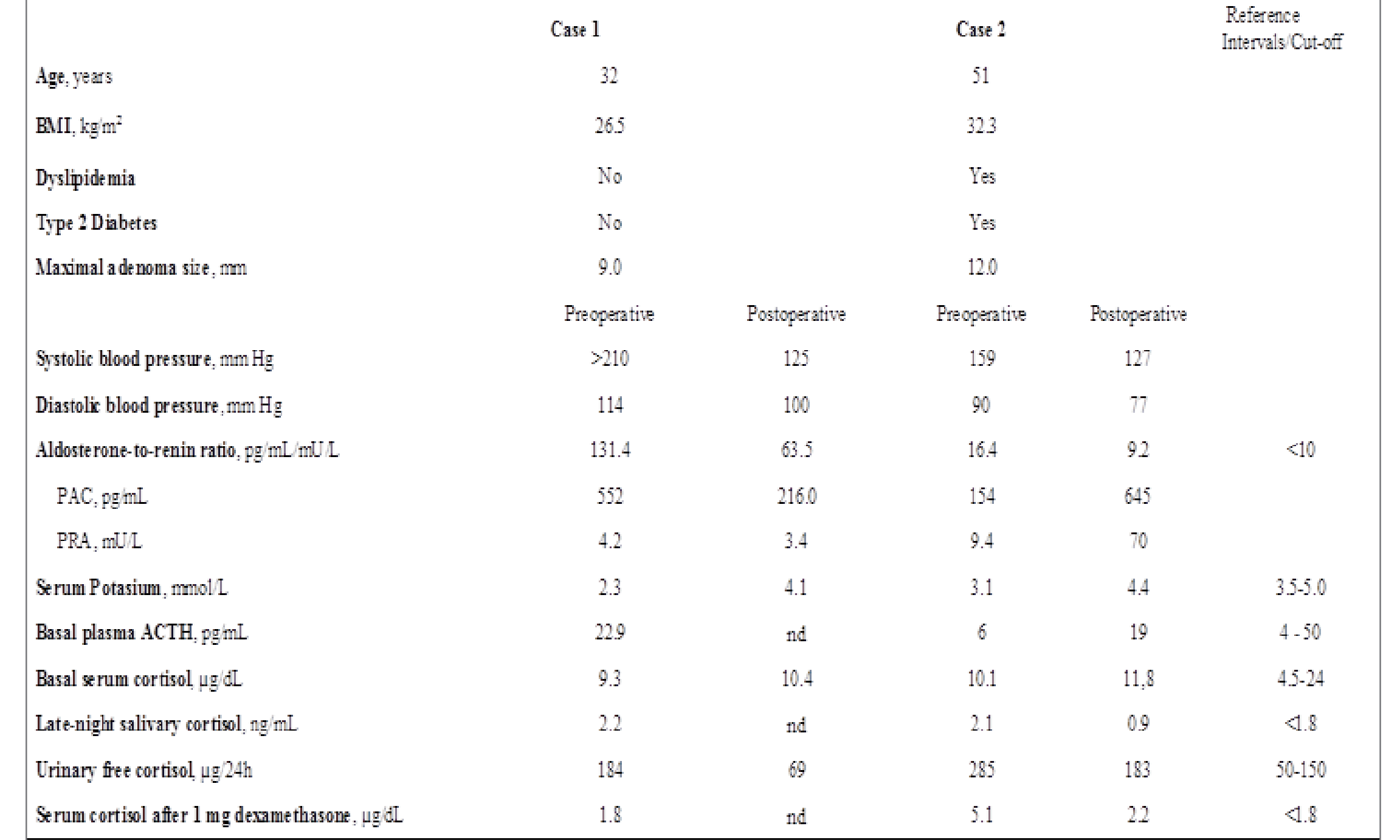

Figure 2: Functional and biochemical characteristics of APAs carrying PRKACA variants

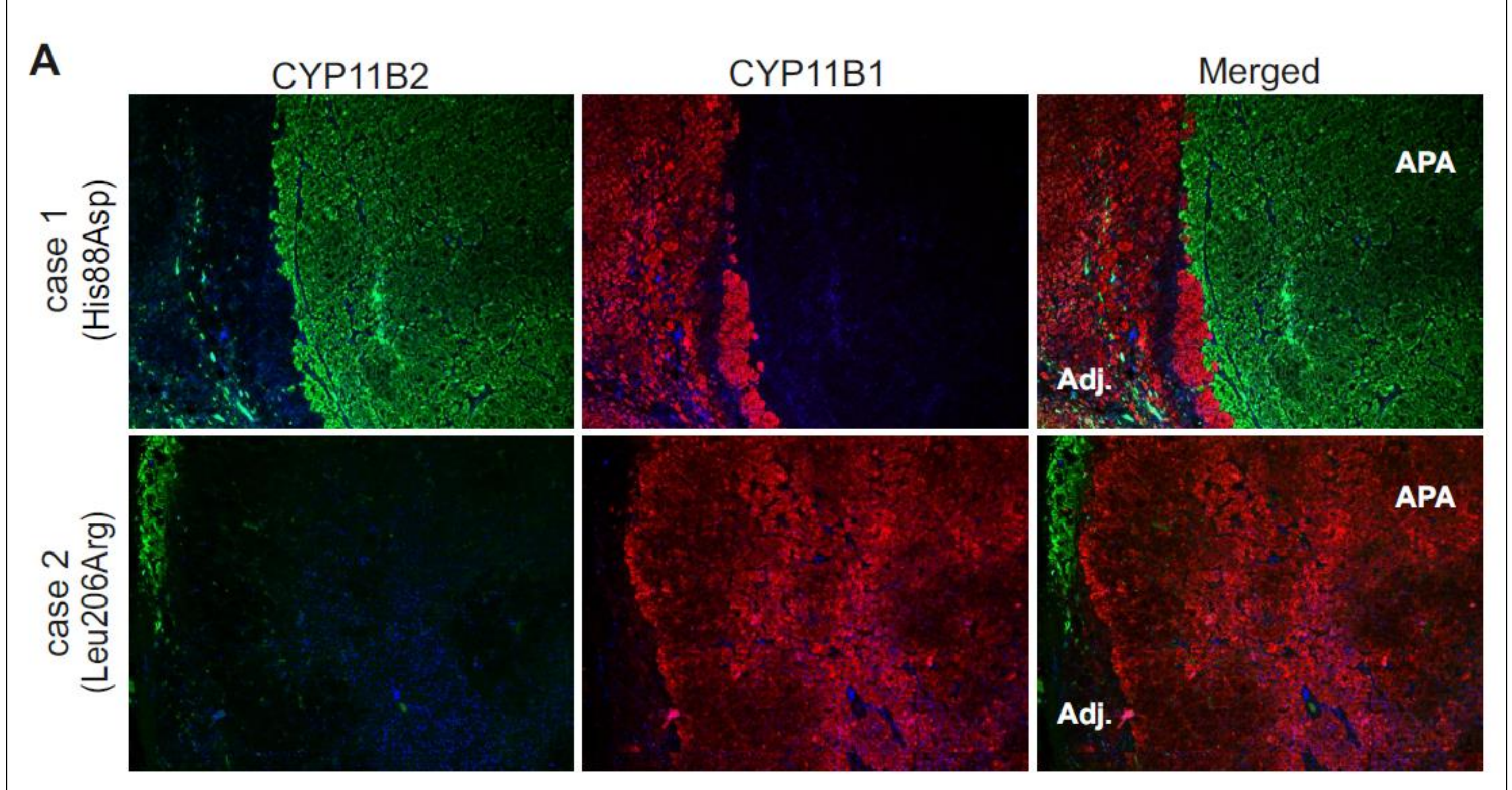

Panel
tissue.

B

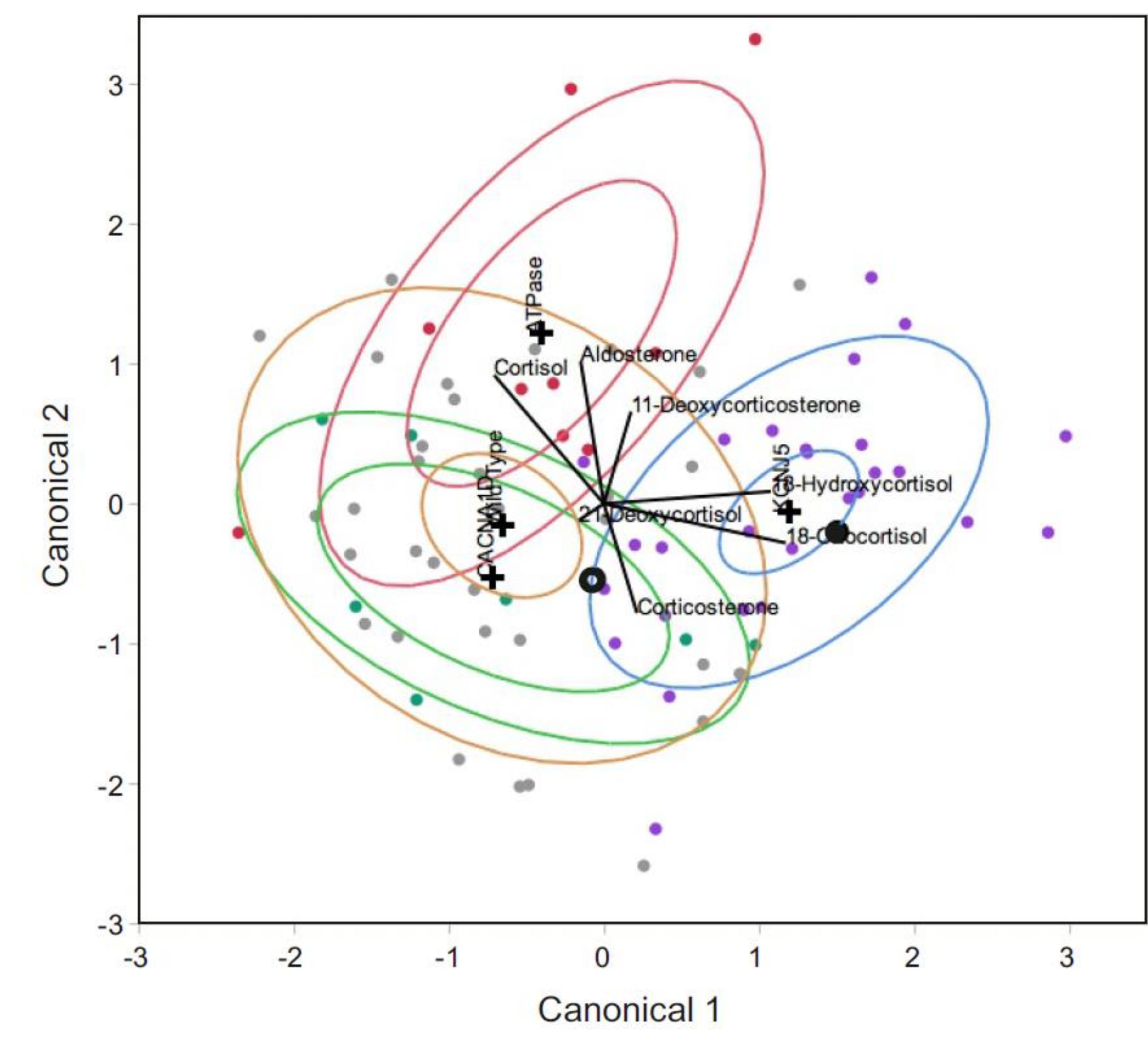

Panel B shows the 20-canonical plot derived from discriminant analysis for plasma concentrations of seven adrenal
steroids (aldosterone, 18--xococortisol, 18-hydroxycortisol, corticosterone, 11-deoxycorticosterone, 21-deoxycortisol and

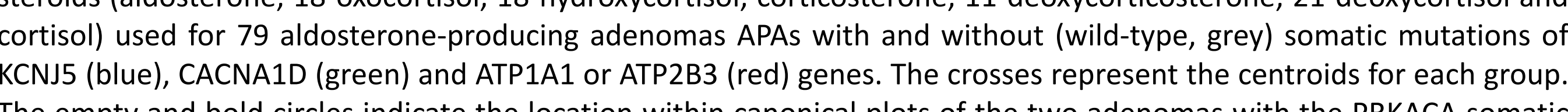

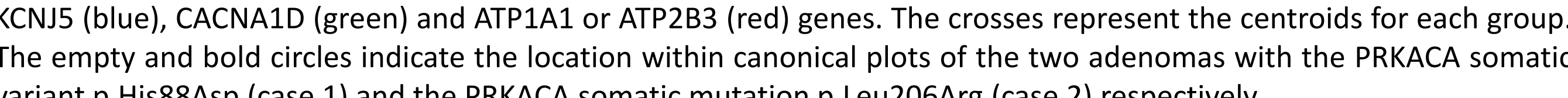

\section{Conclusion}

We describe for the first time PRKACA mutations in two cases of PA patients: a novel PRKACA variant (p.His88Asp) occurring in a case of sudden onset of PA and a PRKACA mutation (p.Leu206Arg) in context of hypokalemic aggravation of long term hypertension. These genetic alterations were not found in a subsequent series of $120 \mathrm{APA}$ and thereby appear to be infrequent events. The molecular basis for co-secretion of aldosterone and cortisol as observed in a subgroup of PA patients remains to be elucidated. 